\title{
Claves para la tutoría virtual
}

\author{
Keys to Virtual Tutoring
}

\section{Jorge Antón-Jornet ${ }^{1}$; Alicia López-Palomera ${ }^{2}$}

En estos tiempos de crisis sanitaria y social, el e-learning supone una oportunidad de formación en un mundo cada vez más globalizado, donde el acceso a internet roza el $60 \%$ de la población mundial, según un informe presentado en 2020 por We Are Social y Hootsuite. Esta modalidad de aprendizaje se afianza como un potente transformador social gracias a su accesibilidad: disponible en cualquier momento, abordable desde cualquier lugar. Asimismo, se consolida como un recurso fundamental para la formación a lo largo de la vida y el aprendizaje en contextos informales.

El e-learning masivo (MOOC, NOOC, SPOOC ${ }^{3}$ ), resultado del auge de los recursos educativos abiertos y del aprendizaje social abierto, iniciado en 1999 por el Instituto Tecnológico de Massachusetts (MIT), supuso una nueva revolución al incorporar otro elemento a la ecuación anterior: llegaba ahora a cualquier persona.

Sin embargo, desde nuestra experiencia y perspectiva, aún faltaba por definirse un agente esencial: el/la tutor/a virtual. Este es un actor fundamental para el acompañamiento y guía de el/la estudiante durante su itinerario formativo y también una necesidad, ya que se estima que solo en torno a un $7 \%$ de los/as estudiantes consigue finalizar un MOOC. (Jordan, 2014). El/la tutor/a virtual incide tanto en el proceso de aprendizaje de los/as estudiantes como en su grado de satisfacción, en la medida en que realiza, como señalara

\footnotetext{
${ }^{1}$ Profesor coordinador de la oficina internacional y coordinador de formación en el IES Salvador Gadea, Aldaia, España; ingeniero civil; jajornet@aulamater.eu.

2 Profesora catedrática de secundaria y coordinadora de proyectos internacionales en el IES Salvador Gadea, Aldaia, España; licenciada en Filología Hispánica; alpalomera@aulamater.eu.

${ }^{3}$ Entendemos como MOOC (Massive Open Online Course) a los cursos masivos abiertos, como NOOC (Nano Open Online Course) a los minicursos masivos abiertos, y como SPOOC (Self-Paced Open Online Course) a los cursos masivos abiertos al ritmo del participante.
} 
REVISTA SABERES EDUCATIVOS, № 5, JULIO-DICIEMBRE 2020

Hernández Madrigal (2007), funciones académicas, organizativas, sociales, orientadoras e incluso técnicas, referida a la plataforma y sus interacciones.

\section{Escenarios para la tutoría virtual}

La educación y la formación a lo largo de la vida pueden darse en escenarios diversos, según los cuales es posible distinguir las categorías de aprendizaje formal (estructurado y certificado), no formal (estructurado) e informal.

En la formación en línea, la tutorización virtual se lleva a cabo fundamentalmente en el ámbito formal. En este, el/la tutor/a virtual debe ser garantía de calidad y eficacia del proceso formativo, y para ello es necesario que se produzca una adaptación de estrategias y métodos, según las características de el/la estudiante meta, los ámbitos y las etapas educativas.

\section{Los desafíos de la tutoría virtual}

Desde hace una década, el autor y la autora del presente artículo venimos desarrollando contenidos y acompañamiento para capacitar al profesorado en el diseño y la realización de proyectos colaborativos internacionales de innovación. Esta labor obliga a una permanente autoevaluación acerca de los ámbitos de actuación de el/la tutor/a creador/a de contenidos y guía para conseguir un óptimo resultado en los/as docentes participantes desde el rol de estudiantes.

Si abordamos esta reflexión sobre la tutorización desde el principio, cabe preguntarse ¿cuál es, en definitiva, la naturaleza de el/la tutor/a? ¿El/la tutor/a nace o se hace?

Es indudable que hay verdad en cualquiera de las respuestas. El/la tutor/a, como toda persona amante de la trasmisión del conocimiento, lleva dentro de sí el germen de la divulgación y la vocación por la enseñanza, y de manera permanente actualiza no solo sus conocimientos, sino también sus estrategias para optimizar la formación de sus 
PUNTO DE VISTA: CLAVES PARA LA TUTORÍA VIRTUAL / ANTÓN-JORNET; LÓPEZ-PALOMERA

tutorandos/as, haciendo verdadera la máxima de Séneca: "Homines dum docent discunt”, ("El hombre enseñando, aprende"). ${ }^{4}$

Este aprendizaje que redunda en aquel que enseña, ocurre de manera natural como parte del propio acompañamiento formativo, pero se complementa conscientemente con una actualización sistemática que garantiza la competencia de el/la formador/a.

El vademécum del tutor, además de su bagaje, se llena de actitudes y estrategias favorecedoras de su cometido, como son la cercanía, la empatía, la disponibilidad y la colaboración con sus pares, puesto que, a partir de ahí, apoya y acompaña al aprendiz; lo motiva, anima, ayuda y facilita su aprendizaje; orienta y guía su formación; retroalimenta sus avances y anima a la mejora desde un enfoque global de la actividad formativa.

Las características del aprendizaje mediante modelos e-learning determinan un protocolo de actuación de la función tutorial, que responde a una jerarquía de acciones encaminadas a guiar a el/la estudiante coherentemente por los contenidos del curso y sus tareas. Responder mensajes privados, resolver dudas, promover reflexiones en los foros colaborativos, revisar y evaluar tareas aportando retroalimentación a el/la estudiante, son estrategias que funcionan y refuerzan la dimensión de los cursos.

Estas estrategias se inscriben en los principales procesos que aborda el/la tutor/a: gestión de datos y contenidos (con hojas de cálculo y plataformas virtuales), curación de contenidos, comunicación con los/as participantes asumiendo la netiqueta ${ }^{5}$ y utilizando herramientas síncronas (chat, videoconferencia) o asíncronas (mensajería, foros), y compartición de los recursos generados con especial atención a la difusión en redes sociales, que garantizan un amplio alcance y la consolidación de una verdadera comunidad virtual de intereses comunes.

En la práctica que se presenta, se utilizan distintos procedimientos para conseguir una necesaria cercanía con el/la estudiante, que lo predisponga para un aprendizaje más eficaz y de mayor recorrido. Entre estos cabe destacar el apoyo en metodologías especialmente motivadoras que enriquecen los modelos e-learning o b-learning, la personalización de

\footnotetext{
${ }^{4}$ Séneca incluye en sus Epístolas Morales VII, 6, esta máxima que afirma que el aprendizaje humano nunca acaba.

${ }^{5}$ Entendemos por netiqueta el conjunto de recomendaciones para el buen uso de la comunicación en la red, tales como las referidas al uso del correo electrónico, las redes sociales, los foros de discusión, los chats, etc.
} 
REVISTA SABERES EDUCATIVOS, № 5, JULIO-DICIEMBRE 2020

los mensajes utilizando siempre el nombre de el/la estudiante desde una delicada cercanía; el estímulo para participar en tareas voluntarias; la promoción y la colaboración en los foros, auténticas salas de debate, reflexión y encuentro; y el planteamiento de tareas colaborativas que se realizan en grupos.

En la línea de vida de cada acción formativa virtual, la complicidad profesional entre tutor/a y estudiante va gestándose a medida que avanzan los módulos y sus tiempos. A lo largo de ella, el estilo de comunicación de el/la tutor/a se hace cada vez más patente. Nuestra "marca", adoptada durante los distintos cursos, se basa en un estilo natural, cercano y sencillo. En ocasiones utiliza el humor discreto, incorpora siempre el nombre del destinatario en los mensajes y manifiesta empatía.

\section{Competencias de un/a tutor/a virtual a través de nuestra experiencia}

La experiencia conseguida a lo largo de los años de capacitación para docentes de forma presencial, semipresencial y no presencial, proporciona a este artículo de reflexión una visión comparada de las necesidades de conjunto y, por ende, de las competencias que precisa un/a tutor/a virtual.

\section{Portafolio digital de un/a tutor/a virtual}

La pregunta primera sobre la función tutorial se centra en si el/a tutor/a debe crear los contenidos, ser su autor/a, o ser un/a guía para contenidos ya creados. Realmente, las labores de la tutorización no precisan necesariamente de las de autoría, aunque cualquier tutor/a debería ser capaz de crear los contenidos que facilita, en virtud de su especialización sobre el tema o área de su labor.

¿Qué competencias pueden ser las necesarias y componen el portafolio de el/la tutor/a virtual? El/la tutor/a virtual debe manejar, sin duda, un amplio abanico de herramientas y recursos digitales, como son:

- Plataformas LMS (Learning Management System) con las que se desarrollan y administran los cursos (Moodle, como referencia, cuando hablamos de plataformas libres). 
PUNTO DE VISTA: CLAVES PARA LA TUTORÍA VIRTUAL / ANTÓN-JORNET; LÓPEZ-PALOMERA

- Herramientas web 2.0: aplicaciones online, herramientas de gestión, de publicación de contenidos, recursos colaborativos, redes sociales.

- Aplicaciones educativas: aplicaciones móviles (Apps), programas informáticos, programas nativos.

- Repositorios de contenidos y recursos.

\section{Figura 1}

Ejemplo de nube de palabras, creado con www.nubedepalabras.es

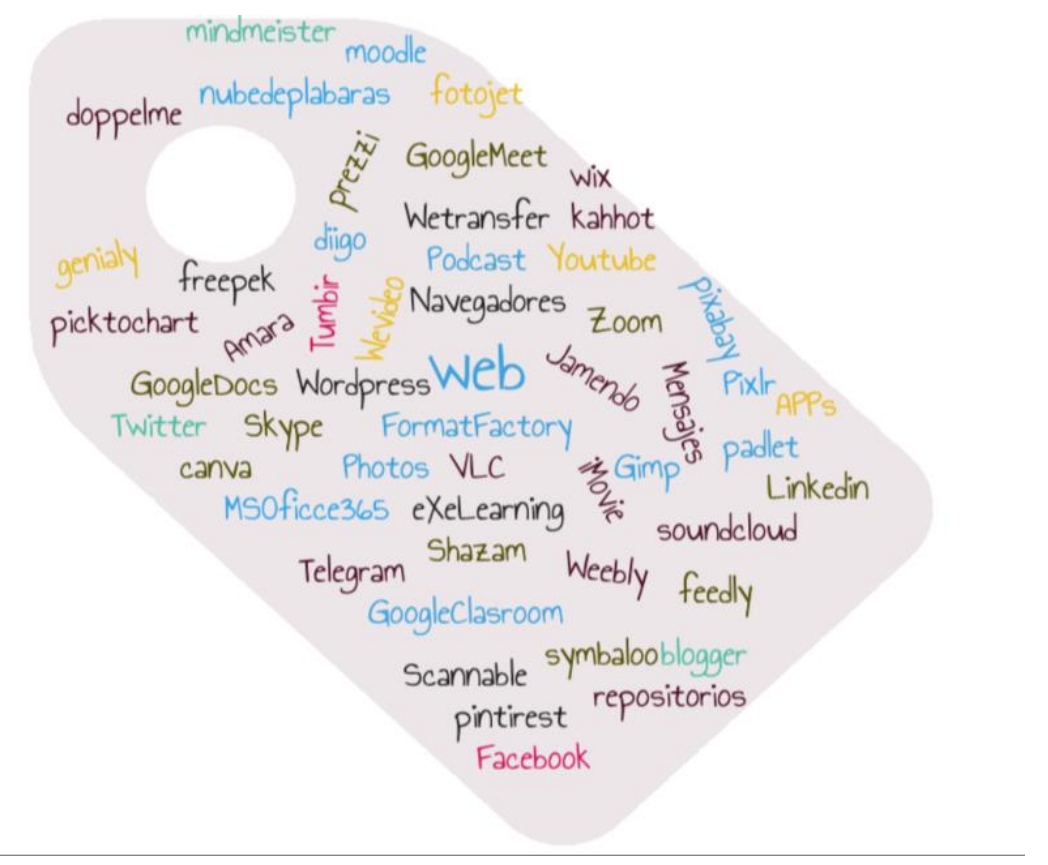

En estos momentos, en los que empieza a estar en auge la web 3.0, con todo tipo de dispositivos conectados, interacción entre aplicaciones, personalización e inteligencia artificial aplicada, el/la tutor/a virtual deberá añadir nuevas utilidades a un portafolio inacabable de competencias digitales.

Es imprescindible establecer, en la formación virtual, rutas formativas coherentes y niveladas, con acceso diferenciado dependiendo del grado de conocimiento previo. Para ello se recomienda configurar un marco de referencia claro, por niveles, basado en el enfoque internacional de marcos de referencia: básico, intermedio y avanzado. 


\section{Diseño significativo}

La responsabilidad primera del/la tutor/a, que puede ser creador/a de contenidos y también facilitador/a de los mismos y guía para los/as estudiantes, es realizar un curso de formación efectivo y que cumpla su propósito original: la experiencia que describe este artículo se enfoca en capacitar a los/as docentes inscritos/as para el diseño, implementación y cierre de un proyecto colaborativo de innovación internacional. Para ello debe proporcionar:

- Contenidos interesantes y de calidad, actualizados, completos y testeados por él/ella mismo/a, pues es imprescindible que haya experimentado aquellos modelos en los que está formando.

- Arquitectura del aprendizaje del itinerario formativo con una correcta agrupación de recursos y herramientas, según el nivel establecido.

- Organización visual perfecta de materiales y recursos en la plataforma virtual que, además, respondan a una categorización coherente y bien estructurada.

- Presentación de materiales estéticamente atractivos y con una línea de diseño y paleta cromática inalterable a lo largo del curso.

- Enfoque de tareas fundamentalmente práctico y dirigido a la realidad del curso, planteando, siempre que sea posible, la creación de recursos significativos que van a ser utilizados por el/la tutorando/a en su diseño de proyecto futuro. Es muy importante que el/la participante sienta que las tareas son (o serán) útiles para él/ella y que no se plantean sobre escenarios irreales o ficticios.

- Estrategias y metodologías innovadoras para estimular la participación y despertar el interés, consiguiendo estudiantes verdaderamente activos/as e ilusionados/as durante el curso.

\section{Aprendizaje adaptativo}

Con todo, para conseguir cumplir los objetivos planteados, es necesario personalizar cada actividad formativa en función de la realidad de las personas inscritas, que son diferentes 
PUNTO DE VISTA: CLAVES PARA LA TUTORÍA VIRTUAL / ANTÓN-JORNET; LÓPEZ-PALOMERA

en cada edición, lo que hace imprescindible adaptar la tutorización a cada contexto determinado por cada comunidad concreta. Para lograr esto, el/la tutor/a se coloca al lado de los/as participantes y facilita su aprendizaje, resolviendo los escollos del camino.

Las principales dificultades detectadas en el desarrollo de los cursos a lo largo del tiempo y los modos que se proponen para su resolución son:

\section{Comienza la apertura del curso y pocos/as participantes han accedido al aula} virtual que, seguramente, les parece neutra y poco cercana. Para aportar cercanía es recomendable presentar el curso con una sesión en línea o, en su defecto, con un breve vídeo mediante el que el/la participante podrá poner voz y cara a el/la tutor/a que va a facilitar su avance en el curso.

2. Pocos/as participantes se conectan en los días de apertura de cada módulo formativo. Como en el caso anterior, puede funcionar iniciar cada módulo formativo con un vídeo en el que la presencia y voz de el/la tutor/a estimulen al avance.

3. Un/a participante plantea una duda en uno de los foros o escribe un mensaje privado para poder continuar su tarea. Ante cualquier duda planteada en mensaje privado o foros, es muy importante ofrecer una respuesta en un plazo de tiempo corto, a ser posible, no más de 24 horas.

4. Se constata poca participación en los foros y los mensajes que hay son aislados. Es recomendable comenzar el curso con alguna tarea de presentación que haya de realizarse en un foro específico y que incluya la interacción o respuesta de las publicaciones de otros/as compañeros/as. Así se consigue que los/as participantes se acostumbren a utilizar esta herramienta de comunicación asíncrona aportando también su potencial colaborativo. Del mismo modo, puede crearse un foro de dudas por cada módulo invitando con interacciones frecuentes a la publicación de mensajes.

\section{Un/a participante manifiesta que no entiende nada y no se ve capaz de} continuar con el curso. Desde una postura cercana y empática, se debe descartar en primer lugar si el problema es de tipo tecnológico, por desconocimiento del manejo de la plataforma virtual. En caso afirmativo, se recomienda de nuevo la lectura de la guía de uso y se resuelven estas primeras cuestiones técnicas. 
REVISTA SABERES EDUCATIVOS, № 5, JULIO-DICIEMBRE 2020

6. Se repiten las mismas dudas o preguntas por medio de mensajes privados. $\mathrm{Se}$ crea un apartado de preguntas frecuentes (FAQ), organizado por categorías y se recomienda su uso.

7. Algunos/as participantes no consiguen realizar bien las tareas, aunque cumplen los plazos. La retroalimentación en la evaluación de las tareas presentadas ha de hacerse con aportes significativos e individualizados, que verdaderamente estén en relación con el trabajo presentado y no sean globales o difícilmente identificables.

8. A pesar de la retroalimentación en una tarea incompleta o mal planteada, el/la estudiante no consigue encontrar el modo de superarla. Resulta especialmente útil grabar un pequeño video tutorial ex profeso, mostrando el escritorio en el ordenador de el/la tutor/a y poniendo voz para la resolución de la duda específica o el error persistente.

9. Determinadas tareas son entregadas en un formato no compatible con el equipo de el/la tutor/a. Dado que una de las labores del/la tutor/a es la de facilitar los contenidos y resolver problemas, es conveniente intentar convertir el archivo para poder acceder a él. Además, esta solución suele ahorrar tiempo y mensajes de ida y vuelta a el/la estudiante para pedir que lo intente él/ella.

10. Algunos/as participantes consideran que la cantidad de información proporcionada en el curso es excesiva. Es importante delimitar muy bien los recursos que son imprescindibles para seguir la formación y aquellos que son materiales de refuerzo o consulta, para una atención más personalizada en función de los intereses del/la tutorando/a.

11. A pesar de todas las estrategias de apoyo y ayuda de el/la tutor/a, algunos/as participantes se encuentran perdidos/as en el curso, que les parece complejo. Se ofrece, con asistencia voluntaria, la realización de videoclases de resolución de dudas. Además, para facilitar que el/la participante elija el curso adecuado, antes de la matriculación, se proporciona un cuestionario que permite saber si los contenidos del curso son adecuados para el nivel de conocimientos previos del/la participante. Esta acción se vincula con el itinerario formativo diseñado para el marco de referencia: básico, intermedio y avanzado. 
PUNTO DE VISTA: CLAVES PARA LA TUTORÍA VIRTUAL / ANTÓN-JORNET; LÓPEZ-PALOMERA

12. En las videoclases los/as participantes tardan en familiarizarse con las herramientas. Se proporciona con antelación una mini-guía de recomendaciones para el manejo de la herramienta de videoconferencia, en formato atractivo, generalmente como infografía, para allanar las siguientes conexiones.

\section{Conclusión}

Considerando estas reflexiones realizadas partir de las experiencias de tutorización de cursos de capacitación de docentes, un/a tutor/a virtual se conforma como una figura poliédrica que acompaña a el/la estudiante en su itinerario de formación, acercando los contenidos, facilitando su comprensión, estimulando el aprendizaje y retroalimentando los productos, para animar a la mejora y consecución de los logros programados como objetivos del curso.

\section{Referencias}

Comisión Europea (2013). Reglamento $N^{o} 1288 / 2013$ del Parlamento Europeo y del Consejo de 11 de diciembre de 2013 por el que se crea el programa «Erasmus+», de educación, formación, juventud y deporte de la Unión y por el que se derogan las Decisiones N 1719/2006/CE, 1720/2006/CE y 1298/2008/CE. Recuperado de: https://op.europa.eu/es/publication-detail/-/publication/9f8f714d-6bfe-11e39afb-01aa75ed71a1/language-es

Hernández Madrigal, P. (2007). Funciones de la tutoría virtual. Recuperado el 27/05/2020 de https://www.calameo.com/books/0023918645f5156f26fb1

Jordan, K. (2014). Initial Trends in Enrolment and Completion of Massive Open Online Courses. International Review of Research in Open and Distance Learning, 15(1).

We Are Social \& Hootsuite. (2020). Digital 2020 Global Digital Overview. Recuperado de $\quad$ https://wearesocial-net.s3-eu-west-1.amazonaws.com/wpcontent/uploads/common/reports/digital-2020/digital-2020-global.pdf 